\title{
VivoSpace: Towards Health Behavior Change Using Social Gaming
}

\author{
Noreen Kamal ${ }^{1}$, Sidney Fels ${ }^{1}$, Michael Blackstock ${ }^{2}$, and Kendall $\mathrm{Ho}^{3}$ \\ ${ }^{1}$ Electrical and Computer Engineering, University of British Columbia, \\ Vancouver, Canada \\ \{noreenk, ssfels\} @ece.ubc.ca \\ ${ }^{2}$ Media and Graphics Interdisciplinary Centre, University of British Columbia, \\ mblackst@magic.ubc.ca \\ ${ }^{3}$ eHealth Strategy Office, University of British Columbia, \\ kendall.ho@ubc.ca
}

\begin{abstract}
Social gaming is now surpassing traditional gaming in terms of the total number of players. We describe our research in creating VivoSpace, an online social network application that applies the rapid uptake of social gaming to the domain of serious games for personal health. Specifically, VivoSpace aims to leverage social gaming to motivate positive health behavior change. A user centered design process has begun for designing VivoSpace based on an initial user inquiry questionnaire that revealed key motivations for using online social networks and users' thoughts on health. Interview feedback of the paper prototypes highlighted reluctance to share particular types of health information and apprehension in logging daily information. However, people were fond of the social aspect of sharing personal health information in the context of group challenges and participating in group health activities.
\end{abstract}

Keywords: VivoSpace, health behavior change, online social networks, serious gaming, user-centered design.

\section{Introduction}

Online social networks have proven that people can be highly motivated to become part of a community activity, participate in group activities, enter personal information regularly and form committed behavior patterns. Social games like Zynga's Farmville ${ }^{1}$ and others are surpassing traditional gaming in terms of ongoing participation [6]. They have sparked an innate need for communication and belonging that has led to a committed user group. Conversely, living a healthy life remains elusive for many. While many people realize the importance of maintaining a healthy lifestyle and often feel that they can live a healthier life, they often have difficulty in managing their health. At a community level, a healthier population can lower healthcare costs; therefore, it is not surprising that many public health initiatives exist that encourages citizens to live more healthy lives [e.g. 4].

\footnotetext{
${ }^{1}$ http: / /www. farmville.com/
} 
There are several theoretical models that articulate the motivations for health behavior change. The health belief model describes the importance of an individual's perceptions on the benefits and barriers to changing their health behavior [7]. Furthermore, the Social Cognitive Theory on health behavior change includes one's perceived self-efficacy, and it includes social factors such as perceived facilitators and impediments to good health behavior $[2,19]$.

These theories provide an interesting vantage point toward understanding the individual and social contributions to health behavior change. However, despite some studies [10,13] it is still unclear how social networks fit into these models. The following research question then emerges: how can an online social network and social games be designed to motivate positive health behavior change? Toward answering this broad question we present the current design and evaluation of VivoSpace, an online social network application that is designed to motivate positive health behavior change.

First we review research that has been conducted with respect to social networks for personal health behavior change. Following this, results from a questionnaire are presented to understand motivation to use social networks and change personal health behavior. We then describe VivoSpace's paper prototype and the interviews we conducted to obtain feedback on our prototypes. Finally, conclusions and future work is discussed based on the described results.

\section{Background and Related Work}

There have been some studies that have looked at how online social networks can be used to motivate health behavior change. A preliminary framework for online social network to motivate health behavior change has been developed [8], called the ABC Framework. The framework identified three main components: individual aspects such as Appeal and self-efficacy, social aspects such as Belonging and influences, and temporal aspects that include Commitment.

The Appeal determinants for motivation to use online social networks include providing and getting information, convenience, entertainment, maintaining interpersonal connectivity, social enhancement and self-discovery $[8,9,15,20]$. The Appeal determinants for motivation to change health behavior include individual incentives, perceived beliefs, perceptions on one's self-efficacy and perceptions about outcomes [8,7]. The Belonging determinants for motivation to use online social networks include social categorization, social connection, sense of belonging, social identity and social comparison $[8,9,3,18]$. The Belonging determinants for motivation to change health behavior include subjective norms, environmental cues and social incentives [8,2,19]. For Commitment, the determinants for use of online social networks are derived from organizational commitment, and they include affective, continuance and normative attachments [8,1]; for motivating health behavior change, there are stages of health behavior change that include pre-contemplation, contemplation, preparation, action and maintenance [8,17]. The ABC Framework will be used to assist the user-centered design process by formulating the points of inquiry. 
Related work indicates that social support through online communities is important to patients managing health problems $[10,16]$. Individual and social aspects have been explored for diabetes patients that are controlling their diet, exercise and medication $[11,12,13]$. In these studies, individual reflection and communication with educators was provided by a mobile application. Furthermore, visualization and reflection of social activity has also been used to improve health outcomes in elderly patients, where visualization of a patient's current social activity increases social behavior [14].

There have also been many commercial products that combine the collection of personal wellness and health data with social supports. This includes the nike+ system $^{2}$, where personal running information can be logged and users can participate in group challenges and discussion forums. Similarly, Ayogo's Healthseeker is a Facebook application that combines one's social network and serious gaming for health management. ${ }^{3}$

\section{Initial User Inquiry}

Initially we used a questionnaire to test the validity of the ABC Framework [8] and then used the framework to provide points of inquiry for the design of the VivoSpace social network application. Our aim is to gain insight into end-user motivation through well-tested models. Online and paper questionnaires were used to obtain feedback on both: 1) their motivations in using online social networks and 2) their motivation in changing health behavior.

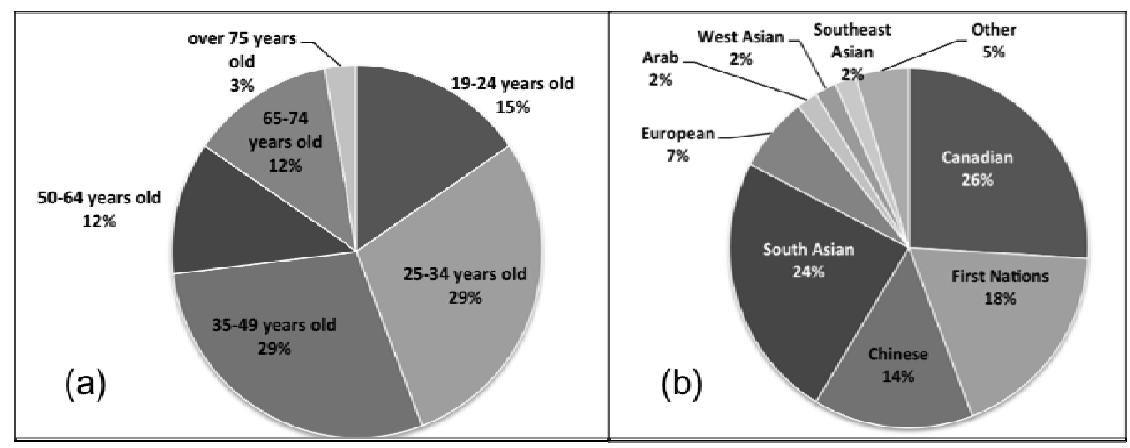

Fig. 1. Demographic distribution for 104 questionnaire respondents. (a) Shows the age distribution, and (b) shows the distribution of ethnic identity.

\subsection{A Look at the Respondents}

104 respondents from a diverse adult population were asked about their motivation to use online social networks and their thoughts on their health behavior. The

\footnotetext{
${ }^{2}$ http: / / nikeplus.com

${ }^{3}$ http: / / ayogo.com/
} 
respondents were $52 \%$ women and $48 \%$ men. The age distribution as shown in figure 1 (a) is quite diverse ranging from 19 to over 74 . The participants' ethnic identity is very diverse with representation from 13 different ethnic groups as shown in figure 1(b). The most represented ethnic groups are Canadian, South Asian, First Nations, and Chinese.

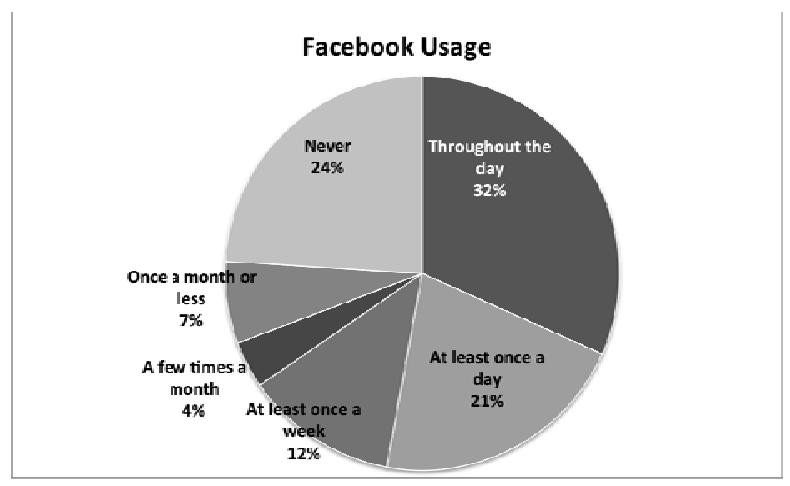

Fig. 2. Frequency of usage for Facebook by questionnaire respondents

The respondents were users of technology to varying degrees. $94 \%$ owned computers with $82 \%$ of all respondents using their computers throughout the day. $53 \%$ of respondents used Facebook at least once a day and only 24\% said they never used Facebook (figure 2). 36\% had used LinkedIn and 29\% had used Twitter. 38\% of respondents had used an online community.

\subsection{Motivation to Use Online Social Networks}

Understanding the motivations to use online social networks is applied in the design of VivoSpace to ensure the social game builds committed behavior. The participants were asked about their motivation for using online social networks or online communities. 86 respondents answered these questions. The points of inquiry are based on the ABC Framework [8], the Uses and Gratification Theory [20,15,9], the Common Bond and Common Identity Theory $[9,18]$, and the Social Identity Theory [3]. Figure 3 shows the key motivators based on individual determinants or the Appeal components of the ABC Framework. Most individual determinants show as being strong motivators, including: maintaining connection with people, easy access to friends, getting information and entertainment. The least favorable motivations reported were to learn more about oneself and to enhance social position.

When the data was stratified by age groups and ethnic groups and one-way ANOVA analysis was conducted, certain ethnic groups were more motivated to use social networks for social enhancement. ANOVA analysis revealed that Chinese and South Asians were motivated to use online social networks for social enhancement more than Canadians $(\mathrm{p}=0.003)$. Table 1 shows the descriptive statistics for these three ethnic groups, where the 5-point Likert scale was numerically defined as 1 is strongly disagree and 5 is strongly agree. 


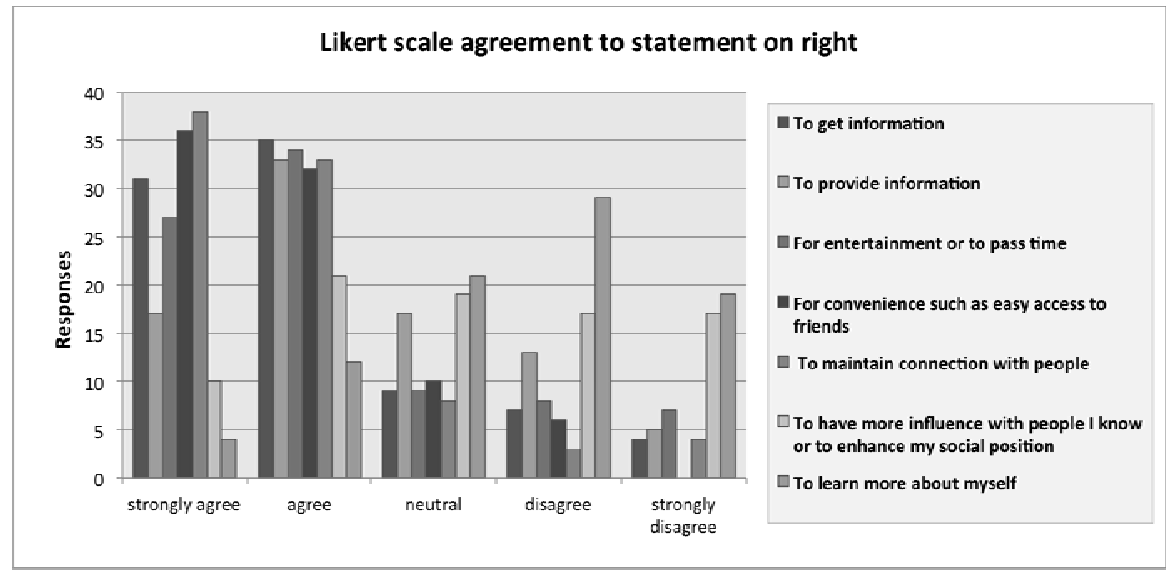

Fig. 3. Responses from 86 respondents for individually based motivation to use online social networks

Table 1. Descriptive statistics for 3 ethnic groups using 5-point Likert scale (1=strongly disagree and $5=$ strongly agree) that resulted in a significant difference are shown here. These responses were for the question "I use online social networks and/or online communities to have more influence with people I know or to enhance my social position"

\begin{tabular}{lccc}
\hline Ethnic Group & $\begin{array}{l}\text { Average } \\
\text { Response }\end{array}$ & $\begin{array}{c}\text { Number of } \\
\text { Responses }\end{array}$ & $\begin{array}{c}\text { Standard } \\
\text { Deviation }\end{array}$ \\
\hline Chinese & 3.53 & 15 & 0.915 \\
South Asian & 3.47 & 15 & 1.246 \\
Canadian & 2.08 & 25 & 1.115 \\
\hline
\end{tabular}

The respondents were also asked about their social motivations to use online social networks. This inquiry was based on the Common Identity and Common Bond Theory $[18,8]$. This is shown in figure 4. Generally, respondents felt stronger about connecting with similar people than belonging to a group or community, but both showed strong motivation. 54\% agreed or strongly agreed that they use online social networks to connect with similar people. $36 \%$ agreed or strongly agreed to using online social networks to belong to a group.

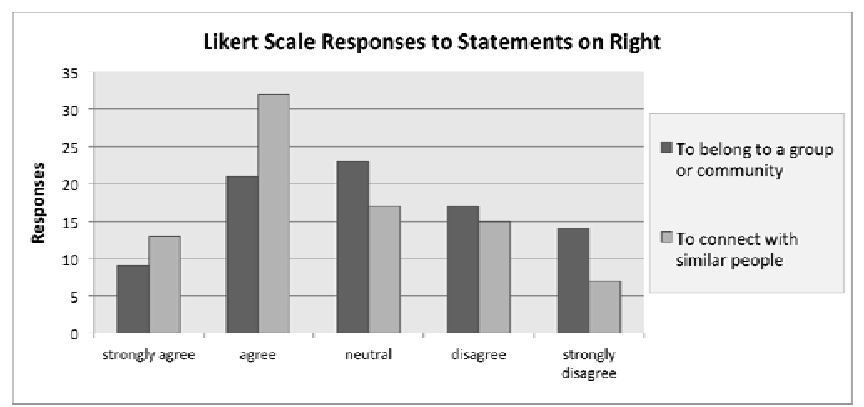

Fig. 4. Responses from 86 respondents for socially based motivation for using online social networks 


\subsection{Thoughts on Health}

102 of the 104 respondents answered the questions inquiring about their thoughts on their health. Figure 5 shows the results. Generally respondents felt that they were living a healthy lifestyle, understanding how to live healthy, eating healthy food and exercising regularly. However, interestingly, the majority of respondents felt that they are capable of living a healthier lifestyle. Most respondents also recognized the social influences on health.

Once again ANOVA analysis was conducted on this data to understand the differences between the age groups and ethnic groups. The ANOVA analysis revealed that there was a difference between the age groups in their perceptions of eating health food $(\mathrm{p}=0.036)$. Respondents aged 65-74 years old (average Likert scale $=4.36$ ) felt that they ate healthy food more than those aged 25-34 years old (average Likert scale $=3.66$ ).

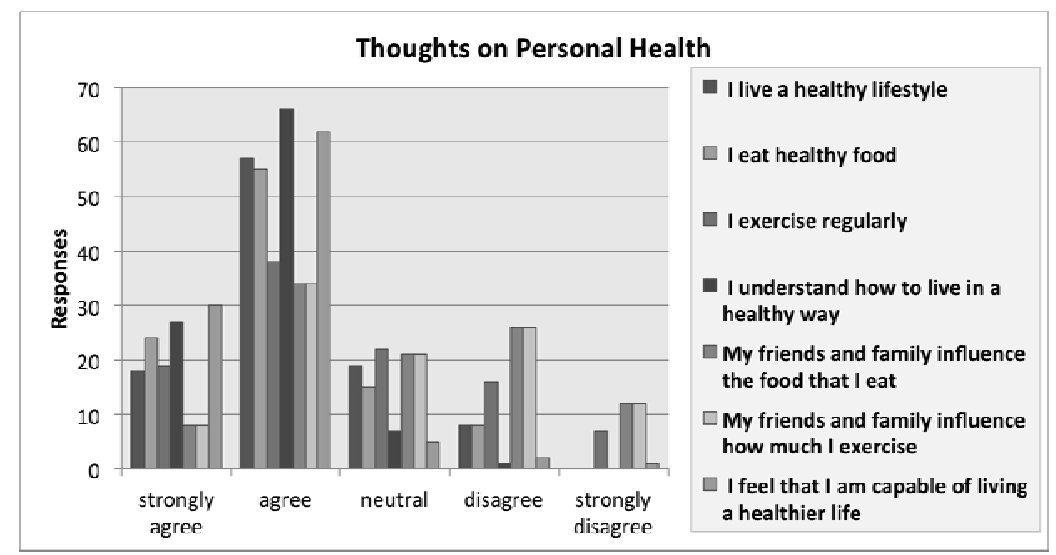

Fig. 5. 102 questionnaire respondents' thoughts around personal health management

\section{VivoSpace Prototype}

Based on the data gathered from the user inquiry and the ABC Framework, which is based on existing theoretical models, we began the design of Vivospace. Initial paper prototypes provide relatively high-fidelity aesthetics to allow us to obtain feedback on both the aesthetic appeal as well as how users feel about its use.

Figures 6 and 7 show 2 of the 14 pages that were developed. The overall design of the paper prototypes included three main frames: the organization pane, the digital asset pane and the content pane. The left pane is the organization pane and it contains items such as an overall scale to see how the user is performing in terms of their health behavior, a calendar that allows the user to enter upcoming events such as a dinner engagement with friends and also has the ability to invite friends to events, a to-do list that will allow the user to manage all their action items whether they are health related or not, and finally a list of the friends or contacts on the VivoSpace 
system. The right pane is a consolidation of the user's digital assets such as music, photos, movies and links that will be connected to their iTunes and/or other digital storage locations either online or on their personal computer. This pane also has connections to online communities that are part of VivoSpace, including a restaurant community where users can rate restaurants and suggest healthy menu items, a recipe community that allow users to share recipes, and a health community to allow discussions around specific health topics. These two panes are meant to build the Appeal dimension of the theoretical framework by linking to digital assets such as music and other informational needs of the user such that health is not separated from other parts the user's life. The central and most prominent pane contains all of the content, including a timeline where users enter their health related activities, a Facebook-style newsfeed and a dashboard.

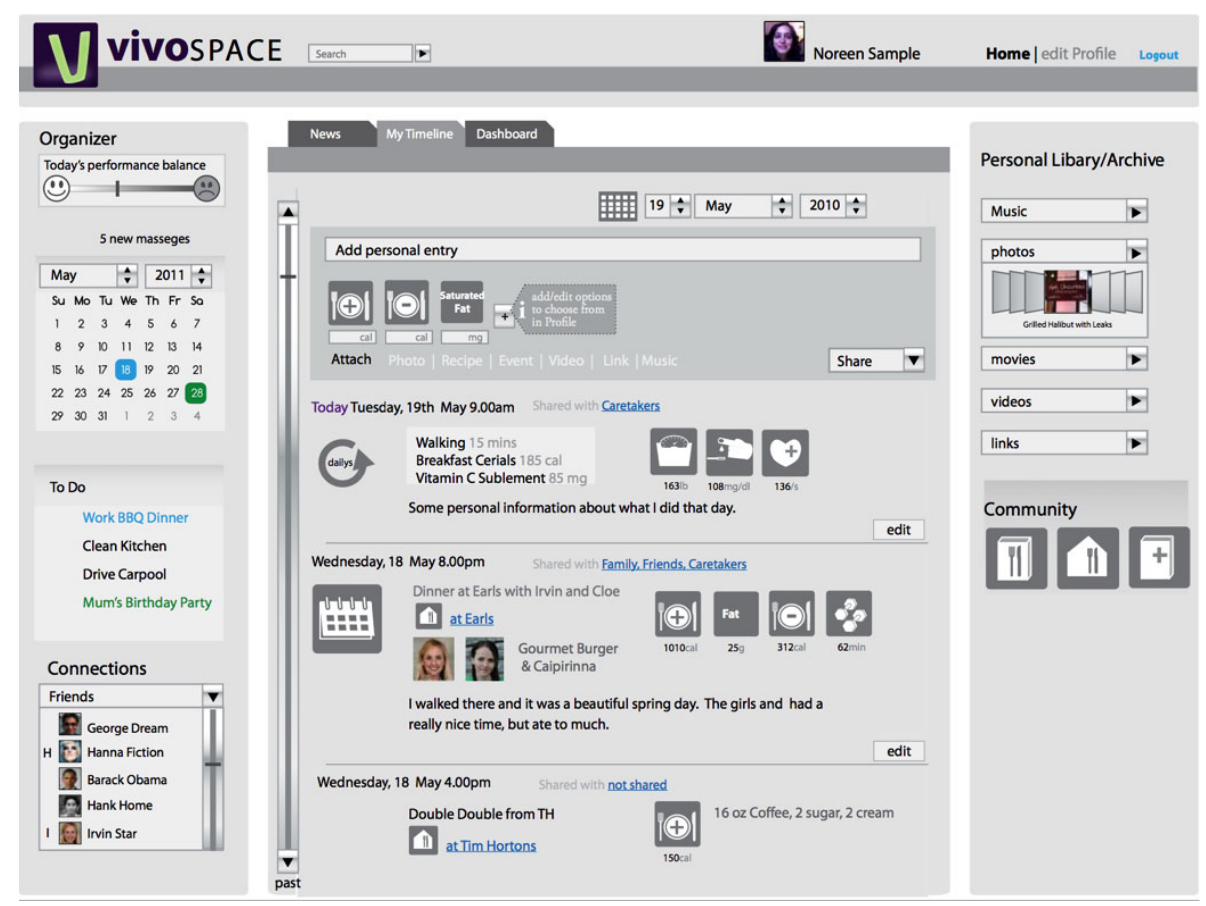

Fig. 6. Timeline is where users are able to log their daily activity and share with any portion of their social network or not share it

The timeline page (figure 6) is where users log their daily health activity. Based on this activity, VivoSpace provides the health information for that activity such as calories and sodium consumed or calories burned. For example, a user can add that they had a specific restaurant meal and a couple of glasses of wine; the system will look up the health related information for this activity such as total calories, amount of sodium and amount of saturated fat, and return that information. This will change the user's perceptions of how their behavior affects their health, which is central to what the theoretical models have said influences health behavior. VivoSpace also 
provides the user with the ability to link a health activity to their social activity; for example, this user had that restaurant meal with two friends who are also on VivoSpace. This will provide the user with feedback on social influences on their health, as there may be certain friends who are good influences, while others are not. This visibility into the user's social determinants should motivate them to change their health behavior as defined by the ABC Framework. Specifically, the Belonging dimension of the theoretical framework to motivate health behavior change includes the subjective norm determinant. The user can also share any of the information in the timeline with all, none or a portion of their social network. The activity for friends can be seen in the Facebook or Twitter-style newsfeed page.

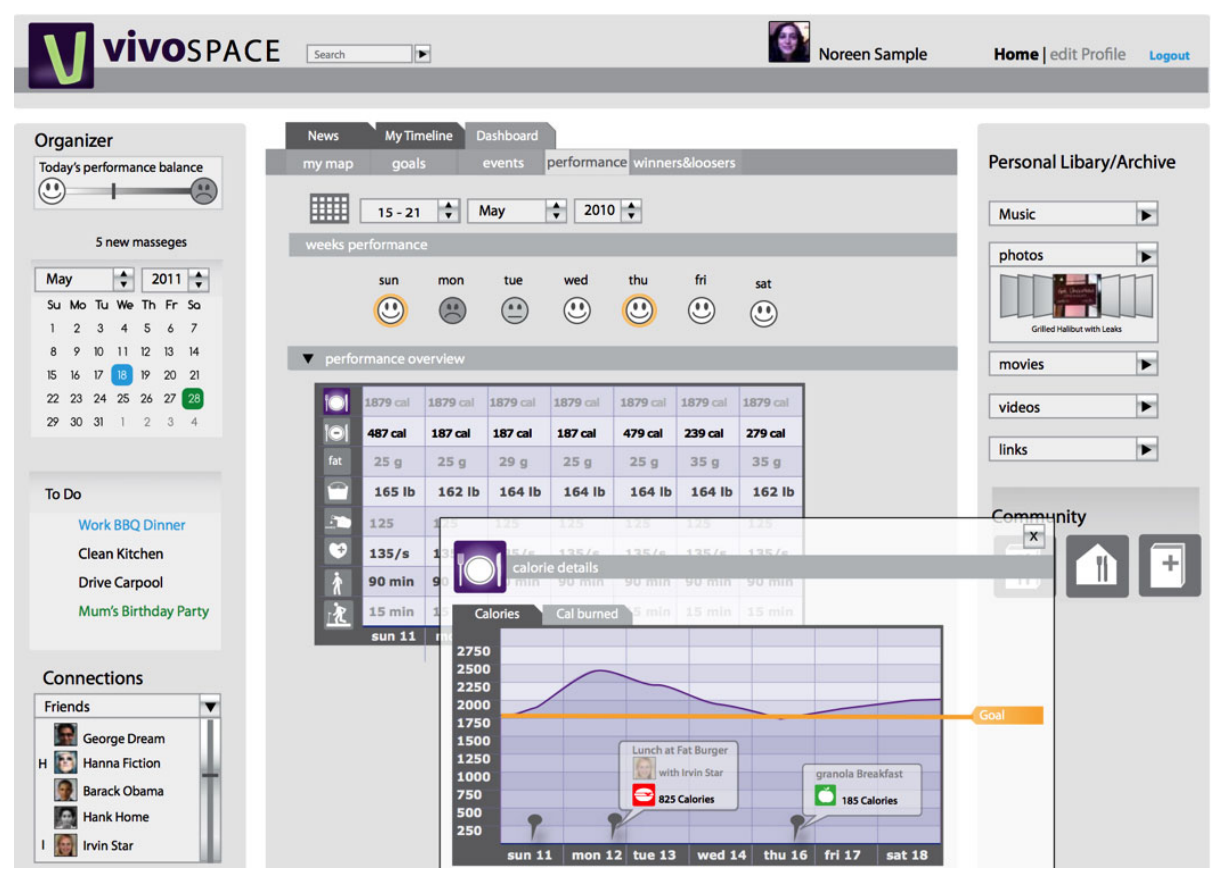

Fig. 7. Dashboard of weekly performance with flags displayed on the graph to show what activities that were logged on that day

Figure 7 shows a health dashboard based on the information that was logged. The user's health performance is shown initially in a narrow channel as happy and sad faces with higher fidelity information provided below. Users have access to a full graphical view of various health variables with annotations of activities that were logged. The "Winners and losers" tab on the dashboard show how the user is performing when compared to their friends. This tab provides a true social gaming experience. The top three and worse performing friends are shown for each day to build social motivation. The "my map" tab provides a geospatial view of ones movements and location of activities. The prototypes also include profile presets, where users can select which health variables they wish to see. Users can also view and select evidence-based seals such as "Diabetes Safe" or "Low in Saturated Fat".. 


\section{Feedback on Prototype}

11 adults (4 women and 7 men) were interviewed to obtain feedback on all 14 pages of VivoSpace's paper prototypes. The participants were regular users of technology and social networks. One participant used social media throughout the day, checking their friend's status updates every few minutes. 9 used either Facebook or Twitter several times everyday. One respondent stopped using Facebook, but continued to use Twitter a few times a week. Figure 8 shows a photograph of all 14 pages laid out during one of the interviews. First, each participant was through the functionality for each page, and then they were asked questions based on the ABC Framework.

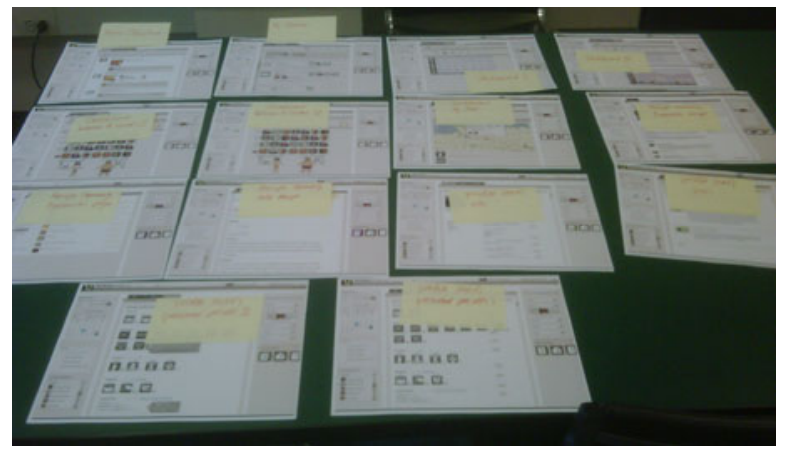

Fig. 8. Photograph of all 14 pages of the paper prototype laid out during the interviews

394 comments about VivoSpace were collected and analyzed. They were coded into 10 categories and then each comment is grouped into related themes [5]. Table 2 shows these 10 categories with the number of comments for each category and the number of themes that emerged in each category.

Table 2. Qualitative analysis of feedback of VivoSpace showing the categories, number of comments in each category and number of themes that emerged in each category.

\begin{tabular}{lcc}
\hline Category & \# of comments & \# of themes \\
\hline Difficulties with VivoSpace & 91 & 20 \\
"Likes" about VivoSpace & 84 & 17 \\
Recommendations & 82 & 23 \\
Dislikes about VivoSpace & 37 & 9 \\
Positive about VivoSpace's ability to influence health & 29 & 7 \\
Positive about social aspects & 21 & 4 \\
Design Aesthetics & 16 & 8 \\
Temporal aspects & 20 & 3 \\
Negative about social aspects & 10 & 7 \\
Explanation of other electronic health products & 4 & 2 \\
\hline
\end{tabular}

Table 3 shows the themes that emerged from the category "difficulties with VivoSpace". The main concern was the perceived difficulty in entering information, as it was felt that inaccurate information would make the system less effective. Privacy concerns were also raised, as it was felt that health information is personal. 
The second most frequently mentioned was concerns around privacy of health information. Specifically, participants voiced concern around whether they would be willing to share unhealthy behavior, and some felt that they would not share health information at all. Many felt that access to the digital assets (personal library) did not fit the scope of Vivospace, and that the prototypes had too much information.

There were many aspects of VivoSpace that participants liked. Table 4 shows the themes that emerged from this category and the number of comments for each theme. The most frequently mentioned was that logging of health information would be helpful, which is at odds the perceived difficulty in logging this information. The dashboard was the page that was most liked, as well as the recipe sharing aspect of the prototype. The participants liked certain design elements such as the personal preset icons. Encouragingly, participants did like some of the social gaming aspects such as the "winners and losers" and the idea of using social networks to track life behavior.

Table 3. Themes emerging from Difficulties with VivoSpace category

\begin{tabular}{ll}
\hline Theme & $\begin{array}{l}\text { \# of } \\
\text { comments }\end{array}$ \\
\hline Too difficult to enter information \& data collection concerns & 20 \\
Privacy: not wanting to share unhealthy habits or any health information & 13 \\
Too much material & 10 \\
Digital assets/personal library does not fit & 7 \\
Lack the motivation to use the system & 4 \\
Not wanting to join another social network & 4 \\
Sees difficulty in adding a recipe & 4 \\
Privacy: willing to share a portion of the post/entry what you are doing but not the nutritional info & 3 \\
Seals are not trustworthy & 2 \\
Only health conscious people would find it enjoyable & 2 \\
Concerned about how recipes are shared & 2 \\
Needs a certain amount of people to be interesting & 2 \\
Better to provide broad guidelines than numbers & 2 \\
Privacy Concerns: Not wanting to share the information & 2 \\
6 other items mentioned once each & \\
\hline
\end{tabular}

Table 4. Themes emerging from Likes about VivoSpace category

\begin{tabular}{lc}
\hline Theme & \# of comments \\
\hline Personal health/life logging would be helpful & 16 \\
Likes dashboard & 16 \\
Likes recipe sharing aspect & 9 \\
Likes the personal presets icons and/or filtering option & 8 \\
Likes winners and losers & 8 \\
Likes the idea of social network to track life behavior & 5 \\
Likes newsfeed / familiar Facebook feel & 4 \\
Likes having all information in one place & 3 \\
VivoSpace would help one to organize one's life & 3 \\
Likes mymap & 3 \\
Likes calendar & 2 \\
6 other items mentioned once each & \\
\hline
\end{tabular}

The participants shared many recommendations to improve VivoSpace (table 5). They were very encouraged by the idea of social gaming and there were numerous suggestions to create challenges to allow groups to assist one in achieving their goals. 
Another recommendation was to make one's health goals more central to the design. The participants were concerned about managing another online social network, so they suggested that VivoSpace should integrate with existing social networks like Facebook and/or Twitter.

Table 5. Themes emerging from Recommendations for VivoSpace category

\begin{tabular}{lc}
\hline Theme & $\begin{array}{c}\text { \# of } \\
\text { comments }\end{array}$ \\
\hline Creation of challenges or groups working together to achieve a goal & 20 \\
Goals need to be more central & 12 \\
Need to integrate with existing social networks and online calendars & 7 \\
Better to allow for customized dashboards and trackers & 7 \\
Needs an incentive system & 6 \\
Need to collect other types of information & 5 \\
Have rankings rather than winners and losers & 3 \\
VivoSpace should remain focused on health information & 2 \\
Create badges / sharing of others achievements & 2 \\
Health information of friends on Winners \& Losers should include other information & 2 \\
Suggestions for dashboard & 2 \\
System needs to provide recommendations \& encouragement on how to live and organize one's life & 2 \\
Need to be able to customize the to-do list and calendar colours & 2 \\
5 other items mentioned once each & \\
\hline
\end{tabular}

\section{Conclusions and Future Work}

The ABC Framework shows strong validity based on inquiry on motivation to use online social networks and change health behavior. Therefore, the ABC Framework was used as points of inquiry for the initial stages of design for VivoSpace. The current prototype design and evaluation of VivoSpace has presented some encouraging results in the creation of a social game for promoting health behavior change. Interview participants were fascinated by the socialization of health information and suggested the creation of challenges and group health activities as motivational features. However, the privacy issues raised still need to be resolved: the next iteration of the design needs to consider how best to create a fun social gaming experience with health information, while providing the user with a sense of control over their privacy.

Based on this feedback, we are currently reworking the design of VivoSpace. The main changes anticipated include: simplicity in its use by allowing user to log aspects of their health; allowance for the creation of challenges that the user can invite specific friends to participate in; making goals central to the design; and showing friends' activities that are most beneficial to their health.

These changes will be incorporated into a medium fidelity prototype that will provide an interactive system. Usability studies will then be conducted to evaluate the medium fidelity prototype. The results from the usability studies will be used to create the next iteration of the system. Specifically, a high fidelity prototype will be developed. This prototype will be used to conduct a field evaluation of VivoSpace to answer the question on how social networks combined with social games can be used to motivate health behavior change. 
Acknowledgements. We thank Wibke Kreft for the design of the paper prototypes, and the Vancouver Aboriginal Friendship Centre for assisting us to recruit First Nations participants for the initial user inquiry questionnaire.

\section{References}

1. Allen, N.J., Meyer, J.P.: The measurement and antecedents of affective, continuance and normative commitment to the organization. Jrn. of Occ. Psych. (63), 1-18 (1990)

2. Bandura, A.: Health promotion by social cognitive means. Health Education Behavior 31(2), 143-164 (2004)

3. Dholakia, U.M., Bagozzi, R.P., Pearo, L.K.: A social influence model of consumer participation in network- and small-group-based virtual communities. Internet J. Research in Marketing 21, 241-263 (2004)

4. Eating Well with Canada's Food Guide, http://www.hc-sc.gc.ca/fn-an/food-guide-aliment/index-eng.php

5. Glaser, B.G., Strauss, A.: Discovery of Grounded Theory: Strategies for Qualitative Research. Sociology Press (1967)

6. Hanluain, D.: Mobile, Social Games Disrupt Gaming Industry. Mobileedia, http: / / www. mobiledia.com/news / 83869.html

7. Janz, N.K., Becker, M.H.: The Health Belief Model: A Decade Later. Health Education Behavior 11(1), 1-47 (1984)

8. Kamal, N., Fels, S., Ho, K.: Online social networks for personal informatics to promote positive health behavior. In: Proc. WSM 2010, pp. 47-52. ACM Press, New York (2010)

9. Lampe, C., Wash, R., Velasquez, A., Ozkaya, E.: Motivations to participate in online communities. In: Proc. CHI 2010, pp. 1927-1919. ACM Press, New York (2010)

10. Maloney-Krichmar, D., Preece, J.: A multilevel analysis of sociability, usability, and community dynamics in an online health community. ACM Trans. on Comput.-Hum. Interact, 201-232 (2005)

11. Mamykina, L., Mynatt, E.D.: Investigating and Supporting Health Management Practices of Individuals with Diabetes. In: Proc. HealthNet 2007, pp. 49-54 (2007)

12. Mamykina, L., Mynatt, E.D., Davidson, P.R., Greenblatt, D.: MAHI: Investigation of Social Scaffolding for Reflective Thinking in Diabetes Management. In: Proc. CHI 2008, pp. 477-486 (2008)

13. Mamykina, L., Miller, A.D., Mynatt, E.D., Greenbalt, D.: Constructing identities through storytelling in diabetes management. In: Proc. CHI 2010, pp. 1203-1212 (2010)

14. Morris, M.: Social Networks as Health Feedback Displays. IEEE Internet Comp. 9(5), 2935 (2005)

15. Papacharissi, Z., Rubin, A.M.: Predictors of internet use. J. Broadcasting \& Electronic Media 44(2), 175-196 (2000)

16. Preece, J.: Online Communities - Designing Usability, Supporting Sociability. John Wiley \& Sons, Chichester (2000)

17. Prochaska, J.O., Velicer, W.F.: The transtheoretical model of health behavior change. American Journal of Health Promotion 12(1), 38-48 (1997)

18. Ren, Y., Kraut, R., Kiesler, S.: Applying common identity and bond theory to design of online communities. Organizational Studies 28(3), 377-408 (2007)

19. Rosenstock, I.M., Victor, J.S., Brecker, M.H.: Social learning theory and the health belief model. Health Education Behavior 15(175), 175-183 (1988)

20. Ruggiero, T.E.: Uses and gratification theory in the 21 st century. Mass Communication \& Society 3(1), 3-37 (2000) 